\title{
Silica Supported Fluoroboric Acid: An Efficient and Reusable Heterogeneous Catalyst for Facile Synthesis of 2-Aliphatic Benzothiazoles, Benzoxazoles, Benzimidazoles and Imidazo[4,5-b]pyridines
}

\author{
Abasaheb V. Patil, Babasaheb P. Bandgar, ${ }^{\dagger}$ and Soo-Hyoung Lee* \\ School of Semiconductor and Chemical Engineering, Nanomaterials Processing Research Center, \\ Chonbuk National University, Jeonju 561-756, Korea. E-mail: shlee66@jbnu.ac.kr \\ ${ }^{\dagger}$ Organic Chemistry Research Laboratory, School of Chemical Sciences, Swami Ramanand Teerth Marathwada University, \\ Vishnupuri, Nanded-431606, India \\ Received November 16, 2009, Accepted February 22, 2010
}

Key Words: $\mathrm{HBF}_{4}-\mathrm{SiO}_{2}$, Benzothiazoles, Benzoxazoles, Benzimidazoles, Imidazo[4,5-b]pyridines

Benzothiazole, benzoxazole, benzimidazole and imidazo $[4,5-b]$ pyridine moieties have attracted a great deal of interest in diverse field of chemistry. ${ }^{1,2}$ These heterocycles have achieved significance in pharmacology as antibacterial, ${ }^{3}$ antiviral, ${ }^{4}$ antifungal, ${ }^{5}$ anticancer, ${ }^{6}$ anticonvulsant ${ }^{7}$ and immunosuppressant agents. ${ }^{8}$

Quite a plethora of methods have been developed for the preparation of these heterocycles including the condensation of carboxylic acids, ${ }^{9}$ acid chlorides, ${ }^{10,11}$ orthoesters, ${ }^{12-14}$ esters, ${ }^{15}$ and aldehydes ${ }^{16-21}$ with $o$-aminophenols, $O$-aminothiophenols and $o$-phenylenediamines, dehydration of $o$-acylaminophenols, ${ }^{22}$ the reaction of $o$-quinones with amines ${ }^{23}$ and Beckmann rearrangement of $o$-acylphenoloximes. ${ }^{24}$

However all of these procedures suffer from one or more of the following drawbacks such as long reaction time, low yield of the products, harsh reaction conditions, use of excess amount of reagents, tedious work up procedures and co-occurrence of several side reactions. In addition, most of the catalysts and reagents are expensive, ${ }^{18,20}$ toxic, air sensitive and non-reusable, as they get destroyed in the reaction work up procedure. Therefore, there is still a need to search for better catalysts that could be superior to the existing ones with regards to toxicity, handling and operational simplicity.

The toxic and volatile nature of many organic solvents used in organic synthesis, particularly chlorinated hydrocarbons have posed serious environmental problems. Therefore, the possibility of performing chemical processes in the absence of solvents (solvent-free conditions) has been attracting more attention. ${ }^{25}$ Organic chemists continue to explore novel synthetic methods involving new reagents and catalysts to carry out chemical transformations. One of these novel synthetic methods is to carry out reactions on the surface of solids. Organic reactions were found to occur efficiently and selectively on the surface of solids. ${ }^{26}$ Even in the absence of new chemistry, a surface reaction may be more desirable than a solution counterpart, because the reaction is more convenient to run, or a high yield of product is attained. In recent years, solid acids and supported reagents ${ }^{27,28}$ have attracted much attention due to their low toxicity, high catalytic activity, moisture and air tolerance, ease of separation, recyclability and relatively lower costs.
In continuation of our attempts to explore the catalytic activity of solid supported fluoroboric acid $\left(\mathrm{HBF}_{4}-\mathrm{SiO}_{2}\right)$ for useful organic transformations under solvent-free conditions, ${ }^{29}$ herein we wish to report $\mathrm{HBF}_{4}-\mathrm{SiO}_{2}(2 \mathrm{~mol} \%)$ catalyzed simple and environmentally benign synthesis of 2-aliphatic benzothiazoles, benzoxazoles, benzimidazoles and imidazo[4,5- $b]$ pyridines from the reaction of $o$-aminothiophenols, $O$-aminophenols, $O$ phenylenediamines and 2,3-diaminopyridines with orthoesters under solvent-free conditions.

\section{Results and Discussion}

The catalyst $\mathrm{HBF}_{4}-\mathrm{SiO}_{2}$ was prepared by using the literature procedure. ${ }^{29}$ Initially, a systematic study was carried out for the catalytic evaluation of $\mathrm{HBF}_{4}-\mathrm{SiO}_{2}$ and examined the effect of solvents under various conditions for the synthesis of 2-methyl benzothiazoles using 1 equivalent of $o$-aminothiophenol with 2 equivalent of triethyl orthoacetate (Scheme 1, Table 1).

The reaction was very slow in absence of catalyst (Table1, entry 1). Next, we optimized the quantity of catalyst at room temperature under solvent-free conditions (Table1, entries 2-4). The reaction was best carried at $2 \mathrm{~mol} \%$ loading of the catalyst (Table 1, entry 4). However, inferior results were obtained with $\mathrm{CH}_{2} \mathrm{Cl}_{2}, \mathrm{CHCl}_{3}, \mathrm{CH}_{3} \mathrm{CN}$ and $\mathrm{CH}_{3} \mathrm{OH}$ as solvents (Table 1, entries 5-8). It was also observed that higher amount of catalyst did not improve the results (Table 1, entry 9). A comparative study to explore the effectiveness of $\mathrm{HBF}_{4}-\mathrm{SiO}_{2}$ over aqueous $\mathrm{HBF}_{4}(40 \%)$ at room temperature under solvent-free conditions (Table 1, entry 10) was also done. The experimental results revealed that aqueous $\mathrm{HBF}_{4}(40 \%)$ afforded only $50 \%$ yield of the corresponding product, while low yields were obtained in presence of only silica as a catalyst (Table 1, entries 11-13).

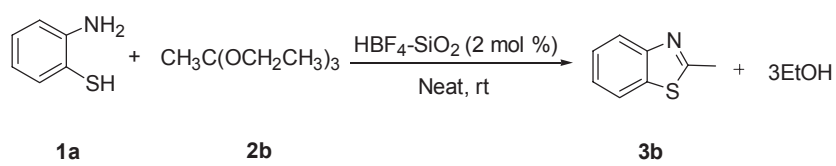

Scheme 1. Synthesis of 2-methylbenzothiazole under various conditions 
Table 1. Investigation of use of solvent and catalyst evaluation in synthesis of 2-methyl benzothiazole.

\begin{tabular}{ccccc}
\hline entry $^{a}$ & solvent & catalyst $(\mathrm{mol} \%)$ & time $(\mathrm{h})$ & yield $^{b}(\%)$ \\
\hline 1 & neat & -------- & 12 & 10 \\
2 & neat & 1 & 1 & 70 \\
3 & neat & 1.5 & 1 & 82 \\
4 & neat & 2 & 1 & 94 \\
5 & $\mathrm{CH}_{2} \mathrm{Cl}_{2}$ & 2 & 1 & 86 \\
6 & $\mathrm{CHCl}_{3}$ & 2 & 1 & 85 \\
7 & $\mathrm{CH}_{2} \mathrm{CN}$ & 2 & 1 & 80 \\
8 & $\mathrm{CH}_{2} \mathrm{OH}$ & 2 & 1 & 82 \\
9 & neat & 3 & 1 & 94 \\
10 & neat & $\mathrm{aq.HBF}_{4}(40 \%)$ & 1 & 50 \\
11 & neat & $\mathrm{SiO}_{2}(100 \mathrm{mg})$ & 1 & 25 \\
12 & neat & $\mathrm{SiO}_{2}(200 \mathrm{mg})$ & 1 & 32 \\
13 & neat & $\mathrm{SiO}_{2}(300 \mathrm{mg})$ & 1 & 38 \\
\hline
\end{tabular}

${ }^{a}$ Reaction condition: $o$-aminothiophenol $(1 \mathrm{mmol})$, triethylorthoacetate (2 mmol); ${ }^{b}$ Isolated yields.

Table 2. Synthesis of 2-aliphatic benzothiazoles, benzoxazoles and benzimidazoles under solvent-free conditions.

\begin{tabular}{|c|c|c|c|c|c|c|c|c|}
\hline \multirow{2}{*}{$\begin{array}{l}\text { product } \\
\text { (3) }\end{array}$} & \multirow{2}{*}{$\mathrm{X}$} & \multirow{2}{*}{$\mathrm{P}$} & \multirow{2}{*}{$\mathrm{R}^{\prime}$} & \multirow{2}{*}{$\begin{array}{c}\text { time } \\
(\mathrm{min})\end{array}$} & \multirow{2}{*}{$\begin{array}{c}\text { yield }^{a, b} \\
(\%)\end{array}$} & \multicolumn{3}{|c|}{ catalyst recycle } \\
\hline & & & & & & I & II & III \\
\hline 3a & S & $\mathrm{H}$ & $\mathrm{H}$ & 45 & $97^{14}$ & 95 & 94 & 92 \\
\hline $3 b$ & S & $\mathrm{H}$ & $\mathrm{CH}_{3}$ & 45 & $94^{14}$ & & & \\
\hline $3 \mathrm{c}$ & S & $\mathrm{H}$ & $\mathrm{CH}_{2} \mathrm{CH}_{3}$ & 45 & $94^{12}$ & & & \\
\hline 3d & S & $\mathrm{H}$ & $\mathrm{CH}_{2} \mathrm{Br}$ & 45 & $95^{33}$ & & & \\
\hline $3 e$ & S & $\mathrm{H}$ & $\mathrm{C} \equiv \mathrm{C}-\mathrm{Si}\left(\mathrm{CH}_{3}\right)_{3}$ & 45 & $84^{34}$ & & & \\
\hline $3 f^{c}$ & $\mathrm{O}$ & $\mathrm{H}$ & $\mathrm{H}$ & 60 & $95^{12}$ & 94 & 92 & 90 \\
\hline $3 g$ & $\mathrm{O}$ & $\mathrm{H}$ & $\mathrm{CH}_{3}$ & 50 & $93^{12}$ & & & \\
\hline $3 \mathrm{~h}$ & $\mathrm{O}$ & $\mathrm{H}$ & $\mathrm{CH}_{2} \mathrm{CH}_{3}$ & 50 & $94^{12}$ & & & \\
\hline $3 \mathbf{i}$ & $\mathrm{O}$ & $\mathrm{H}$ & $\mathrm{CH}_{2} \mathrm{Br}$ & 50 & $92^{33}$ & & & \\
\hline $3 \mathbf{j}$ & $\mathrm{O}$ & $\mathrm{Cl}$ & $\mathrm{CH}_{3}$ & 50 & $92^{24}$ & & & \\
\hline $3 k$ & $\mathrm{O}$ & $\mathrm{CH}_{3}$ & $\mathrm{CH}_{3}$ & 50 & $93^{24}$ & & & \\
\hline 31 & $\mathrm{O}$ & $\mathrm{CH}_{3}$ & $\mathrm{CH}_{2} \mathrm{CH}_{3}$ & 50 & $94^{24}$ & & & \\
\hline $3 \mathbf{m}^{d}$ & $\mathrm{NH}$ & $\mathrm{H}$ & $\mathrm{H}$ & 50 & $94^{14}$ & 92 & 90 & 90 \\
\hline $3 \mathbf{n}^{d}$ & $\mathrm{NH}$ & $\mathrm{H}$ & $\mathrm{CH}_{3}$ & 50 & $90^{32}$ & & & \\
\hline $30^{d}$ & NH & $\mathrm{H}$ & $\mathrm{CH}_{2} \mathrm{CH}_{3}$ & 50 & $94^{31}$ & & & \\
\hline $3 \mathbf{p}^{d}$ & $\mathrm{NH}$ & $\mathrm{H}$ & $\mathrm{CH}_{2} \mathrm{Br}$ & 50 & $91^{33}$ & & & \\
\hline $3 \mathbf{q}^{d}$ & $\mathrm{NH}$ & $\mathrm{CH}_{3}$ & $\mathrm{CH}_{3}$ & 50 & $92^{30}$ & & & \\
\hline $3 \mathbf{r}^{d}$ & NH & $\mathrm{CH}_{3}$ & $\mathrm{CH}_{2} \mathrm{CH}_{3}$ & 50 & $91^{30}$ & & & \\
\hline
\end{tabular}

${ }^{a}$ Products were identified by comparison of their physical and spectral data with those of authentic samples. ${ }^{b}$ Isolated yields. ${ }^{c}$ The reaction was performed at $60{ }^{\circ} \mathrm{C}$. ${ }^{d}$ The reaction was performed at $80{ }^{\circ} \mathrm{C}$ using 3 equivalents of orthoester.

The high activity of $\mathrm{HBF}_{4}-\mathrm{SiO}_{2}$ may be attributed to the larger surface area and better selectivity.

The generality and the applicability of this method was further examined for the synthesis of benzoxazoles and benzimidazoles from various orthoesters under solvent-free conditions (Scheme 2) and the corresponding products were obtained in high to excellent yields (Scheme 2, Table 2).

The results showed that 2 -substituted benzoxazoles were efficiently and rapidly formed under mild reaction conditions at room temperature (Table 2, entries 3g-3I), whereas unsub-

$$
\underbrace{\mathrm{RH}^{\prime} \mathrm{C}\left(\mathrm{OCH}_{2} \mathrm{CH}_{3}\right)_{3} \frac{\mathrm{HBF}_{4}-\mathrm{SiO}_{2}(2 \mathrm{~mol} \%)}{\text { Neat }}}_{X+1}
$$

Scheme 2. Synthesis of 2-aliphatic benzothiazoles, benzoxazoles and benzimidazoles

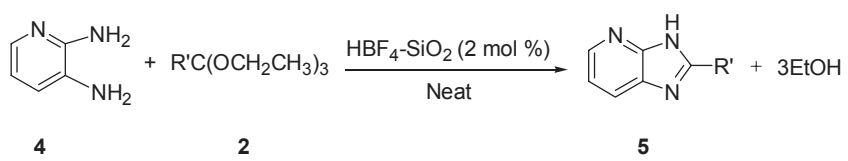

\begin{tabular}{|c|c|c|c|c|}
\hline entry & $\begin{array}{c}\text { catalyst } \\
{[\mathrm{mol} \%] \text { (solvent) }}\end{array}$ & $\begin{array}{l}\text { catalyst } \\
\text { loading }\end{array}$ & $\begin{array}{c}\text { time } \\
(\min )[\mathrm{h}]\end{array}$ & $\begin{array}{l}\text { yield } \\
(\%)\end{array}$ \\
\hline 1 & {$[\mathrm{Hbim}] \mathrm{BF}_{4}$} & stoichiometry & $(10-25)$ & $80-95^{11}$ \\
\hline 2 & {$\left[\mathrm{bbim} \mathrm{BF}_{4}\right.$} & stoichiometry & $(40-120)$ & $79-94^{11}$ \\
\hline 3 & $\begin{array}{l}\mathrm{K}_{2} \mathrm{CO}_{3} \\
\text { (N-methyl pyridine) }\end{array}$ & [5 mol \%] & $(30-45)$ & $50-95^{15}$ \\
\hline 4 & $\begin{array}{l}\mathrm{CrCl}_{2}: \mathrm{Mn}: \mathrm{TMSCl} \\
\text { (DMF) }\end{array}$ & 0.25 eq: 6 eq: 4 eq & {$[12-18]$} & $47-76^{16}$ \\
\hline 5 & Oxone (DMF) & $0.65 \mathrm{eq}$ & {$[0.5-24]$} & $58-90^{17}$ \\
\hline 6 & $\mathrm{Yb}(\mathrm{OTf})_{3}$ & [5 mol \%] & $(30)$ & $66-99^{18}$ \\
\hline 7 & $\mathrm{I}_{2}-\mathrm{KI}$ & 1 eq: 0.25 eq & $(30-50)$ & $65-95^{19}$ \\
\hline 8 & $\operatorname{In}(\mathrm{OTf})_{3}$ & [5 mol \%] & (30) & $74-95^{20}$ \\
\hline 9 & $\mathrm{Na}_{2} \mathrm{~S}_{2} \mathrm{O}_{5}$ & stoichiometry & $(30)$ & $75-94^{21}$ \\
\hline 10 & $\mathrm{HBF}_{4}-\mathrm{SiO}_{2}$ (Neat) & [2 $\mathrm{mol} \%]$ & $(45-60)$ & $84-97$ \\
\hline
\end{tabular}

Scheme 3. Synthesis of 2-aliphatic imidazo[4,5-b]pyridines

Table 3. Comparison of efficiency of $\mathrm{HBF}_{4}-\mathrm{SiO}_{2}$ against recently reported catalyst for synthesis of benzothiazoles, benzoxazoles and benzimidazoles

stituted benzoxazole at 2- position needed slight heating at $60{ }^{\circ} \mathrm{C}$ (Table 2, entry 3f). The reactions of $o$-phenylenediamine with orthoesters were also investigated and found that $o$-phenylenediamines reacted rapidly with orthoesters in presence of $2 \mathrm{~mol} \%$ of $\mathrm{HBF}_{4}-\mathrm{SiO}_{2}$ at $80{ }^{\circ} \mathrm{C}$ under solvent-free conditions. It was also noticed that increase in the proportion of the orthoester to $o$-phenylenediamine $(3: 1)$ produced corresponding benzimidazoles in high yields (Table 2, entries $\mathbf{3 m - 3 r}$ ).

While comparing the effect of catalyst for the synthesis of benzothiazoles, benzoxazoles and bezimidazoles (Table 3), we found that $\mathrm{HBF}_{4}-\mathrm{SiO}_{2}$ was more effective than recently reported catalysts in terms of the amount of catalyst used and corresponding yields under solvent-free conditions.

Encouraged by the above results, the present protocol was extended for the synthesis of imidazo[4,5-b]pyridines using 2 , 3-diaminopyridine and orthoesters under solvent free conditions (Scheme 3). The reactions were performed at higher temperature $\left(80^{\circ} \mathrm{C}\right)$ and corresponding imidazo[4,5-b]pyridines were isolated in good yields (Table 4, entries 5a-5c).

The advantage of the use of heterogeneous catalyst for this transformation is the ease of catalyst separation. When the catalyst reaction was completed, $\mathrm{HBF}_{4}-\mathrm{SiO}_{2}$ could be recovered 
Table 4. Synthesis of 2-aliphatic imidazo[4,5-b]pyridines under solvent free conditions

\begin{tabular}{cccc}
\hline product $(5)^{a}$ & $\mathrm{R}^{\prime}$ & time (min.) & yield (\%) \\
\hline $\mathbf{5 a}$ & $\mathrm{H}$ & 30 & $70^{35}$ \\
$\mathbf{5 b}$ & $\mathrm{CH}_{3}$ & 30 & $85^{35}$ \\
$\mathbf{5} \mathbf{c}$ & $\mathrm{CH}_{2} \mathrm{CH}_{3}$ & 30 & $82^{35}$ \\
\hline
\end{tabular}

${ }^{a}$ All reactions were performed at $80{ }^{\circ} \mathrm{C}$.

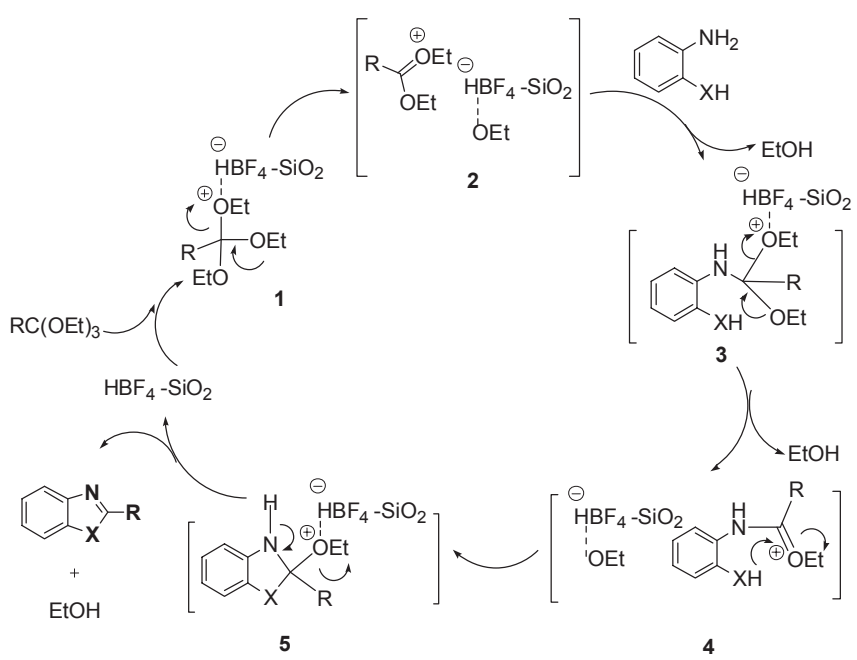

Scheme 4. Plausible mechanism for the $\mathrm{HBF}_{4}-\mathrm{SiO}_{2}$ catalyzed synthesis of benzothiazoles, benzoxazoles and benzimidazoles

conveniently form the reaction mixture through filtration and subsequent washing with ethyl acetate. The recovered catalyst was activated by heating at $80{ }^{\circ} \mathrm{C}$ under vacuum for 2 hours and reused thrice consecutively through a series of sequential reactions of triethylorthformate with $O$-aminothiophenols, $O$ aminophenols, and $o$-phenylenediamines giving excellent yields without substantial loss of reactivity (Table 2, product 3a, 3f and $\mathbf{3 m}$ respectively).

The actual mechanism of the reaction is unclear. However a plausible explanation is that, the orthoester is first activated by $\mathrm{HBF}_{4}-\mathrm{SiO}_{2}$ to give 1 and then $\mathbf{2}$. Further, 2-substituted aniline attacks $\mathbf{2}$, affording $\mathbf{3}$, which is then activated by catalyst yielding 4. Cyclization of $\mathbf{4}$ gives $\mathbf{5}$ which in turn is activated by the catalyst to afford the final corresponding product together with third molecule of EtOH and release of $\mathrm{HBF}_{4}-\mathrm{SiO}_{2}$ for the next catalytic cycle (Scheme 4).

\section{Conclusions}

In conclusion, we have developed a simple, efficient, inexpensive and green synthesis of 2-aliphatic benzothiazoles, benzoxazoles, benzimidazoles and imidazo[4,5- $b]$ pyridines using catalytic amount of $\mathrm{HBF}_{4}-\mathrm{SiO}_{2}$ under solvent-free conditions, where the catalyst can be recovered and reused. In addition, non aqueous easy work up, least catalyst loading ( 2 mol \%), higher yields of product, non toxicity of the catalyst, cost effective, highly efficient conversion make this method eco-friendly, environmentally benign and a valid contribution to the existing process in the field of these heterocyclic synthesis.

\section{Experimental Section}

All the chemicals were analytical grade reagents and were used as obtained from Aldrich Co. without further purification. Melting points were measured using Buchi R-535 apparatus and are uncorrected. The IR spectra were recorded on JASCO Fourier transform (FT)-IR. ${ }^{1} \mathrm{H}$ NMR spectra were recorded using a JEOL FT-NMR (400 MHz) spectrometer using TMS as an internal standard. The structures of all the products were confirmed by ${ }^{13} \mathrm{C}$ NMR, ${ }^{1} \mathrm{H}$ NMR and IR data and comparison with authentic samples prepared according to the literature methods.

Preparation of the catalyst. The catalyst was prepared by following literature procedure. ${ }^{29} \mathrm{HBF}_{4}$ ( $3.3 \mathrm{gm}$ as a $40 \%$ aqueous solution) was added to the suspension of silica gel (26.7 gm, 230 - $400 \mathrm{mesh})$ in diethyl ether $(80 \mathrm{~mL})$. The mixture was concentrated and the residue was dried under vacuum at $100{ }^{\circ} \mathrm{C}$ for 72 hours to yield $\mathrm{HBF}_{4}-\mathrm{SiO}_{2}\left(0.5 \mathrm{mmol}_{\text {of }} \mathrm{HBF}_{4} \mathrm{gm}^{-1}\right)$ as a free flowing powder.

General procedure for synthesis of 2-methylbenzothiazole. A mixture of triethyl orthoacetate $(10 \mathrm{mmol}, 2 \mathrm{eq}$.) and $o$-aminothiophenol ( $5 \mathrm{mmol}, 1 \mathrm{eq}$.) was stirred at room temperature in presence of catalytic amount of $\mathrm{HBF}_{4}-\mathrm{SiO}_{2}(200 \mathrm{mg}, 0.05 \mathrm{mmol}$, $2 \mathrm{~mol} \%$ ) for an appropriate time (Table 2). After completion of the reaction (TLC), the reaction mixture was diluted with $30 \mathrm{~mL}$ ethyl acetate and filtered through a plug of cotton. The cotton plug was washed with ethyl acetate $(10 \mathrm{~mL} \times 3)$. The combined ethyl acetate layer was concentrated under reduced pressure to afford the crude product which was purified further by column chromatography ( $n$-hexane:Ethyl acetate 7:3) as an oil (yield 94\%).

Physical and spectral data of selected products.

2-(Bromomethyl)-1,3-benzothiazole (3d): Pale yellow solid; $\operatorname{mp} 49$ - $50{ }^{\circ} \mathrm{C}$; IR (KBr): $3035,2920,1654,1460,971,826 \mathrm{~cm}^{-1}$; ${ }^{1} \mathrm{H}$ NMR (400 MHz, $\mathrm{CDCl}_{3}$ ): $\delta$ 8.18-8.15 (m, 2H), 7.52-7.49 $(\mathrm{m}, 2 \mathrm{H}), 4.61(\mathrm{~s}, 2 \mathrm{H}) ;{ }^{13} \mathrm{C}$ NMR $\left(100 \mathrm{MHz}, \mathrm{CDCl}_{3}\right): \delta 169.6$, 153.8, 134.7, 127.2, 126.0, 122.9, 121.3, 36.7; EIMS m/z 229 $\left(\mathrm{M}^{+}\right)$; Anal. Calcd. For $\mathrm{C}_{8} \mathrm{H}_{6} \mathrm{NSBr}$ C, 42.12; H, 2.65; N, 6.14. Found: C, 42.10; H, 2.66; N, 6.16.

2-(2-Trimethylsilylethynyl)-1,3-benzothiazole (3e): Viscous oil; IR (Neat): 3040, 2940, 1640, 1450, 1250, $950 \mathrm{~cm}^{-1} ;{ }^{1} \mathrm{H}$ NMR (400 MHz, $\left.\mathrm{CDCl}_{3}\right): \delta 8.15-8.12(\mathrm{~m}, 2 \mathrm{H}), 7.52-7.48(\mathrm{~m}$, 2H), $0.4(\mathrm{~s}, 9 \mathrm{H}) ;{ }^{13} \mathrm{C} \mathrm{NMR}\left(100 \mathrm{MHz}, \mathrm{CDCl}_{3}\right): \delta 158.2,154.5$, 133.8, 127.0, 125.6, 123.0, 122.3, 90.6, 72.7; Anal. Calcd. For $\mathrm{C}_{12} \mathrm{H}_{13} \mathrm{NSSi}$ : C, 62.28; H, 5.66; N, 6.05. Found: C, 62.30; H, $5.65 ; \mathrm{N}, 6.06$.

2-(Bromomethyl)-1,3-benzoxazole (3i): Solid powder; mp 35 - $37^{\circ} \mathrm{C}$; IR (KBr): $3018,2950,1642,1594,1435,1117,1035$, $812 \mathrm{~cm}^{-1} ;{ }^{1} \mathrm{H}$ NMR (400 MHz, DMSO- $\left.d_{6}\right): \delta 7.78-7.75(\mathrm{~m}, 2 \mathrm{H})$, 7.46-7.42 (m, 2H), 4.91 (s, 2H); ${ }^{13} \mathrm{C}$ NMR (100 MHz, DMSO$\left.d_{6}\right)$ : $\delta$ 162.0, 149.6, 141.4, 127.4, 124.7, 121.7, 111.6, 36.5; Anal. Calcd. For $\mathrm{C}_{8} \mathrm{H}_{6} \mathrm{NOBr}$ : C, 45.31; H, 2.85; N, 6.60. Found: C, 45.30; H, 2.86; N, 6.62.

2-(Bromomethyl)-1,3-benzimidazole (3p): Pale yellow solid; mp 59 - $61{ }^{\circ} \mathrm{C}$; IR (KBr): 3264, 3175, 3028, 2962, 2825, 1650, $1470,973 \mathrm{~cm}^{-1}$; ${ }^{1} \mathrm{H}$ NMR (400 MHz, DMSO- $\left.d_{6}\right): \delta 10.2(\mathrm{~s}$, $1 \mathrm{H}, \mathrm{NH}), 7.53-7.56(\mathrm{~m}, 2 \mathrm{H}), 7.21-7.19(\mathrm{~m}, 2 \mathrm{H}), 4.51$ (s, 2H); ${ }^{13} \mathrm{C}$ NMR (100 MHz, DMSO- $\left.d_{6}\right): \delta 155.6,139.8,123.5,116.0$, 
33.5; Anal. Calcd. For $\mathrm{C}_{8} \mathrm{H}_{7} \mathrm{~N}_{2} \mathrm{Br}$ : C, 45.52; H, 3.34; N, 13.28 . Found: C, 45.50; H, 3.35; N, 13.26.

2-Methylimidazo[4,5-b]pyridine (5b): Yellow solid powder; mp 189 - $190{ }^{\circ} \mathrm{C}$; IR (KBr): 3178, 2962, 2918, $1615 \mathrm{~cm}^{-1} ;{ }^{1} \mathrm{H}$ NMR (300 MHz, $\left.\mathrm{CDCl}_{3}\right): \delta 13.7$ (s, br, $\left.1 \mathrm{H}, \mathrm{NH}\right), 8.4-8.2(\mathrm{~m}$, 1H), 8.1-8.0 (m, 1H), 7.25-7.15 (m, 1H), 2.75 (s, 3H); Anal. Calcd. For $\mathrm{C}_{7} \mathrm{H}_{7} \mathrm{~N} 3$ : C, 63.14; H, 5.30; N, 31.56. Found: C, $63.10 ; \mathrm{H}, 5.35 ; \mathrm{N}, 31.51$.

Acknowledgments. This research was supported by the Pioneer Research Center Program through the National Research Foundation of Korea funded by the Ministry of Education, Science and Technology (Contract No. 2008-05103).

\section{References}

1. Denny, W. A.; Revacastle, G. W.; Baguley, B. J. Med. Chem. 1990, $33,814$.

2. (a) Israel, M.; Day, A. R. J. Org. Chem. 1959, 24, 1455 and references cited therein. (b) Kassidy, P. E. Thermally Stable Polymers; Marcell Dekkar: New York, 1980.

3. Yildiz, I. O.; Yalcin, I.; Akisener, E.; Ucarturk, N. Eur. J. Med. Chem. 2004, 39, 291.

4. Song, X.; Vig, B. S.; Lorenzi, P. L.; Drach, J. C.; Townsend, L. B.; Amidon, G. L. J. Med. Chem. 2005, 48, 1274.

5. Yamato, M. J. Pharm. Soc. Jpn. 1992, 112, 81.

6. Kumar, D.; Jacob, M. R.; Reynolds, M. B.; Karvin, S. M. Bioorg. Med. Chem. 2002, 10, 3997.

7. Benazzouz, A.; Boraud, T.; Dubedat, P.; Boireau, A.; Stutzmann, J. M.; Gross, C. Eur. J. Pharmacol. 1995, 284, 299.

8. Grimmett, M. R.; Katritzky, A. R.; Rees, C. W.; Sceiven, E. F. V.; Comprehensive Heterocyclic Chemistry II, Vol 3; Elsevier Science Ltd: Oxford, 1996.

9. Alcalde, E.; Dinares, I.; Perez-Garcia, L.; Roca, T. Synthesis 1992, 395.

10. Pottorf, R. S.; Chadha, N. K.; Katkevics, M.; Ozola, V.; Suna, E.; Ghane, H.; Regberg, T.; Player, M. R. Tetrahedron Lett. 2003, 44, 175.

11. Nadaf, R. N.; Siddiqui, S. A.; Daniel, T.; Lahoti, R. J.; Srinivasan, K. V. J. Mol. Catal. A: Chem. 2004, 214, 155.

12. Jenkins, G. L.; Knevel, A. M.; Davis, C. S. J. Org. Chem. 1961, 26, 274.

13. Doise, M.; Dennin, F.; Blondeau, D.; Sliwa, H. Tetrahetron Lett.
$1990,31,1155$.

14. Villemin, D.; Hammadi, M; Martin, B. Synthetic Commun. 1996, 26, 2895.

15. Chakraborti, A. K.; Rudrawar, S.; Kaur, G.; Sharma, L. Synlett 2004, 1533.

16. Hari, A.; Karan, C.; Rodridues, W. C.; Miller, B. L. J. Org. Chem. 2001, 66, 991.

17. Beaulieu, P. L.; Hache, B.; Von Moss, E. Synthesis 2003, 1683.

18. Curini, M.; Epifano, F.; Montanari, F.; Rosati, O.; Taccone, S. Synlett 2004, 1832.

19. Gogoi, P.; Konwar, D. Tetrahedron Lett. 2006, 47, 79.

20. Trivedi, R.; De, S. K.; Gibbs, R. A. J. Mol. Catal. A: Chem. 2006, 245,8 .

21. Navarrete-Vazquez, G.; Moreno-Diaz, H.; Aguirre-Crespo, F.; Leon-Revara, I.; Villalobos-Molina, R.; Munoz-Muniz, O.; Estrada-Soto, S. Bioorg. Med. Chem. Lett. 2006, 16, 4169.

22. Boyd, G. V.; Katritzky, A. R.; Rees, C. W.; Potts, K. T. Comprehensive Heterocyclic Chemistry; Pregamon Press: New York, 1984; p 177.

23. Bu, X. Z.; Huang, Z. S.; Zhang, M.; Ma, L.; Xiao, G. W.; Gu, L. Q. Tetrahedron Lett. 2001, 42, 5737.

24. Bhaval, B. M.; Mayabhate, S. P.; Likhite, A. P.; Deshmukh, A. R. A. S. Synthetic Commun. 1995, 25, 3315.

25. Tundo, D.; Anastas, P. T.; Green Chemistry: Theory and Practice; Oxford University Press: Oxford, 1998.

26. Toda, F. Acc. Chem. Res. 1995, $28,480$.

27. Chakraborti, A. K.; Gulhane, R. Chem. Commun. 2003, 1896.

28. Hajipoor, A. R.; Abidi, H.; Rooho, A. E. J. Org. Chem. 2003, 68, 4553.

29. (a) Bandgar, B. P.; Patil, A. V.; Kamble, V. T. Arkivoc 2007, 16, 252 and references cited therein. (b) Bandgar, B. P.; Patil, A. V.; Kamble, V. T.; Totre, J. V. J. Mol. Catal. A: Chem. 2007, 273, 114. (c) Bandgar, B. P.; Patil, A. V.; Kamble, V. T.; Chavan, O. S. Cat. Commun. 2007, 8, 1065. (d) Bandgar, B. P.; Patil, A. V.; Chavan, O. S. J. Mol. Catal. A: Chem. 2006, 256, 99.

30. Raban, M.; Chang, H.; Craine, L.; Hortelano, E. J. Org. Chem. 1985, 50, 2205.

31. Wang, H.; Partch, R. E.; Li, Y. J. Org. Chem. 1997, 62, 5222.

32. Wang, L.; Sheng, J.; Tian, H.; Qian, C. Synthetic Commun. 2004, 34,4265 .

33. Joshi, A. A.; Vishwanathan, C. L. Bioorg. Med. Chem. Lett. 2006, 16,2613

34. Kumar, D.; David, W. M.; Kervin, S. M. Bioorg. Med. Chem. Lett. 2001, 11, 2971

35. Heravi, M. M.; Montazeri, N.; Rahmizadeh, M.; Bakavoli, M.; Ghassemzadeh, M. J. Chem. Res. (S) 2000, 584. 